Proceedings of the Edinburgh Mathematical Society (2006) 49, 39-52 (C)

DOI:10.1017/S0013091502000810 Printed in the United Kingdom

\title{
THE POLYNOMIAL NUMERICAL INDEX OF A BANACH SPACE
}

\author{
YUN SUNG CHOI ${ }^{1}$, DOMINGO GARCIA ${ }^{2}$, \\ SUNG GUEN KIM ${ }^{3}$ AND MANUEL MAESTRE ${ }^{2}$ \\ ${ }^{1}$ Department of Mathematics, POSTECH, Pohang 790-784, \\ South Korea (mathchoi@euclid.postech.ac.kr) \\ ${ }^{2}$ Departamento de Análisis Matemático, Universidad de Valencia, \\ Doctor Moliner 50, 46100 Burjasot, Valencia, Spain \\ (domingo.garcia@uv.es; manuel.maestre@uv.es) \\ ${ }^{3}$ Department of Mathematics, Kyungpook National University, \\ Taegu 702-701, South Korea (sgk317@knu.ac.kr)
}

(Received 22 August 2002)

Abstract In this paper, we introduce the polynomial numerical index of order $k$ of a Banach space, generalizing to $k$-homogeneous polynomials the 'classical' numerical index defined by Lumer in the 1970s for linear operators. We also prove some results. Let $k$ be a positive integer. We then have the following:

(i) $n^{(k)}(C(K))=1$ for every scattered compact space $K$.

(ii) The inequality $n^{(k)}(E) \geqslant k^{k /(1-k)}$ for every complex Banach space $E$ and the constant $k^{k /(1-k)}$ is sharp.

(iii) The inequalities for every Banach space $E$.

$$
n^{(k)}(E) \leqslant n^{(k-1)}(E) \leqslant \frac{k^{(k+(1 /(k-1)))}}{(k-1)^{k-1}} n^{(k)}(E)
$$

(iv) The relation between the polynomial numerical index of $c_{0}, l_{1}, l_{\infty}$ sums of Banach spaces and the infimum of the polynomial numerical indices of them.

(v) The relation between the polynomial numerical index of the space $C(K, E)$ and the polynomial numerical index of $E$.

(vi) The inequality $n^{(k)}\left(E^{* *}\right) \leqslant n^{(k)}(E)$ for every Banach space $E$.

Finally, some results about the numerical radius of multilinear maps and homogeneous polynomials on $C(K)$ and the disc algebra are given.

Keywords: polynomial numerical index; numerical radius; Aron-Berner extension; homogeneous polynomials; Banach spaces

2000 Mathematics subject classification: Primary 46A22; 46G20

Secondary 46G25

\section{Introduction}

Let $E$ and $F$ be real or complex Banach spaces. We write $\stackrel{\circ}{B}_{E}, B_{E}$ and $S_{E}$ for the open unit ball, the closed unit ball and the unit sphere of $E$, respectively. The dual space of 
$E$ is denoted by $E^{*}$. Let $k \in \mathbb{N}$. We let $\mathcal{L}\left({ }^{k} E: F\right)$ denote the Banach space of continuous $k$-linear mappings of $E^{k}:=E \times \cdots \times E$ into $F$, endowed with the norm

$$
\|A\|=\sup \left\{\left\|A\left(x_{1}, \ldots, x_{k}\right)\right\|: x_{j} \in B_{E}, j=1, \ldots, k\right\} .
$$

A mapping $P: E \rightarrow F$ is called a continuous $k$-homogeneous polynomial if there is an $A \in \mathcal{L}\left({ }^{k} E: F\right)$ such that $P(x)=A(x, \ldots, x)$ for all $x \in E$. Each such $P$ has a unique associated continuous symmetric $k$-linear map $\check{P}$ of $E^{k}$ into $F$. We let $\mathcal{P}\left({ }^{k} E: F\right)$ denote the Banach space of continuous $k$-homogeneous polynomials of $E$ into $F$, endowed with the polynomial norm $\|P\|=\sup _{x \in B_{E}}\|P(x)\|$. When $F$ is the scalar field $\mathbb{R}$ or $\mathbb{C}$, we denote this space by $\mathcal{P}\left({ }^{k} E\right)$. Note that $\mathcal{P}\left({ }^{1} E: E\right)=\mathcal{L}\left({ }^{1} E: E\right)$ is the space of bounded linear operators on $E$. (See $[\mathbf{1 0}]$ for a general background on the theory of polynomials on an infinite-dimensional Banach space.) Let

$$
\Pi(E)=\left\{\left(x, x^{*}\right): x \in S_{E}, x^{*} \in S_{E^{*}}, x^{*}(x)=1\right\} .
$$

For each $P \in \mathcal{P}\left({ }^{k} E: E\right)$, the numerical range of $P$ is the subset $V(P)$ of the scalar field defined by

$$
V(P)=\left\{x^{*}(P x):\left(x, x^{*}\right) \in \Pi(E)\right\} .
$$

In [5] the numerical radius of $P$ is given by

$$
v(P)=\sup \{|\lambda|: \lambda \in V(P)\}
$$

and the numerical radius of a homogeneous polynomial on some classes of Banach spaces was computed. It is clear that $v$ is a seminorm on $\mathcal{P}\left({ }^{k} E: E\right)$ and $v(P) \leqslant\|P\|$ for every $P \in \mathcal{P}\left({ }^{k} E: E\right)$. We introduce the polynomial numerical index of order $k$ of a Banach space, generalizing to $k$-homogeneous polynomials the 'classical' numerical index defined by Lumer in the 1970s for linear operators. It is natural to consider the polynomial numerical index of order $k$ of the space $E$, namely the constant $n^{(k)}(E)$ defined by

$$
n^{(k)}(E)=\inf \left\{v(P): P \in S_{\mathcal{P}\left({ }^{k} E: E\right)}\right\} .
$$

Equivalently, $n^{(k)}(E)$ is the greatest constant $c \geqslant 0$ such that $c\|P\| \leqslant v(P)$ for every $P \in \mathcal{P}\left({ }^{k} E: E\right)$. Note that $0 \leqslant n^{(k)}(E) \leqslant 1$, and $n^{(k)}(E)>0$ if and only if $v$ and $\|\cdot\|$ are equivalent norms on $\mathcal{P}\left({ }^{k} E: E\right)$. It is obvious that if $E_{1}, E_{2}$ are isometrically isomorphic Banach spaces, then $n^{(k)}\left(E_{1}\right)=n^{(k)}\left(E_{2}\right)$.

The concept of the numerical index (in our terminology, the polynomial numerical index of order 1) was first suggested by Lumer [17]. He gave a theory of the numerical range or bounded linear operators on a Banach space. This is a very successful generalization of the classical theory, in which only Hilbert spaces are considered. At that time, it was known that a Hilbert space of dimension greater than 1 has numerical index $\frac{1}{2}$ in the complex case and 0 in the real case. Several years later, Duncan et al. [11] proved that $L$-spaces and $M$-spaces have numerical index 1 . McGregor [18] obtained necessary and sufficient conditions such that a finite-dimensional normed space has numerical index 1. The disc algebra is another example of a Banach space with numerical index 1 [8, Theorem 3.3]. Crabb et al. [7] investigated some extremal problems in the theory of numerical 
ranges. Recently, Lopez et al. [16] investigated necessary conditions for a real Banach space to have numerical index 1. Martin and Paya [19] studied the numerical index of vector-valued function spaces. For general information and background on numerical ranges we refer to the books by Bonsall and Duncan $[\mathbf{3}, \mathbf{4}]$. Further developments in the Hilbert space case can be found in $[\mathbf{1 3}]$.

In $\S 2$ of this paper we prove the following results. Let $k$ be a positive integer. Then we have the following:

(i) $n^{(k)}(C(K))=1$ for every positive integer $k$ and every scattered compact space $K$.

(ii) The inequality $n^{(k)}(E) \geqslant k^{k /(1-k)}$ for every complex Banach space $E$ and the constant $k^{k /(1-k)}$ is sharp.

(iii) The inequalities

$$
n^{(k)}(E) \leqslant n^{(k-1)}(E) \leqslant \frac{k^{(k+(1 /(k-1)))}}{(k-1)^{k-1}} n^{(k)}(E)
$$

for every Banach space $E$.

(iv) The relation between the polynomial numerical index of $c_{0}, l_{1}, l_{\infty}$ sums of Banach spaces and the infimum of the polynomial numerical indices of them.

(v) The relation between the polynomial numerical index of the space $C(K, E)$ and the polynomial numerical index of $E$.

(vi) The inequality $n^{(k)}\left(E^{* *}\right) \leqslant n^{(k)}(E)$ for every Banach space $E$.

In $\S 3$ some results about the numerical radius of multilinear maps and homogeneous polynomials on $C(K)$ and the disc algebra are given.

\section{Properties of the polynomial numerical index of order $k$}

It was proved in [5, Theorem 3.1(ii)] that $n^{(k)}\left(c_{0}\right)=n^{(k)}(c)=n^{(k)}\left(l_{\infty}\right)=1$ for every positive integer $k$, where $c$ is the Banach space of convergent sequences in $\mathbb{C}$.

Given a Banach space $E$, we denote by $\mathcal{A}\left(B_{E}\right)$ the Banach space of all functions $f: B_{E} \rightarrow \mathbb{C}$ which are holomorphic on $\stackrel{\circ}{B}_{E}$ and uniformly continuous on $B_{E}$, endowed with the supremum norm. Recall that a mapping $P$ is said to be a continuous polynomial on $E$ if it can be represented as a sum

$$
P=P_{0}+P_{1}+\cdots+P_{m}
$$

where $P_{j} \in \mathcal{P}\left({ }^{j} E\right)$ for $j=0, \ldots, m$. The vector space of all continuous polynomials on $P$ is always a dense subspace of $\mathcal{A}\left(B_{E}\right)$.

Lemma 2.1 (see Theorem 3.3 in [6]). Let $K$ be a scattered compact Hausdorff space. If $T$ is an element of $\mathcal{A}\left(B_{C(K)}\right)$, then

$$
\|T\|=\sup \left\{|T(f)|: f \in \operatorname{ext} B_{C(K)}\right\},
$$

where ext $B_{C(K)}$ is the set of all extreme points of $B_{C(K)}$. 
Theorem 2.2. Let $K$ be a scattered compact space. For every positive integer $k$, we have $n^{(k)}(C(K))=1$.

Proof. It suffices to show that $\|P\|=v(P)$ for every $P \in \mathcal{P}\left({ }^{k} C(K): C(K)\right)$. Let $P \in$ $\mathcal{P}\left({ }^{k} C(K): C(K)\right)$. Let $\varepsilon>0$ be given. We can choose $f_{0} \in B_{C(K)}$ and $t_{0} \in K$ such that $\left|P\left(f_{0}\right)\left(t_{0}\right)\right|>\|P\|-\varepsilon$. Define a continuous $k$-homogeneous polynomial $Q: C(K) \rightarrow \mathbb{C}$ by $Q(f)=P(f)\left(t_{0}\right)(f \in C(K))$. By Lemma 2.1 there exists $g_{0} \in \operatorname{ext} B_{C(K)}$ such that $\left|Q\left(g_{0}\right)\right|>\sup _{f \in B_{C(K)}}|Q(f)|-\varepsilon$. Then $\left|g_{0}(t)\right|=1$ for every $t \in K$. It follows that

$$
\begin{aligned}
\|P\|-2 \varepsilon & <\left|P\left(f_{0}\right)\left(t_{0}\right)\right|-\varepsilon \leqslant \sup _{f \in B_{C(K)}}\left|P(f)\left(t_{0}\right)\right|-\varepsilon \\
& =\sup _{f \in B_{C(K)}}|Q(f)|-\varepsilon<\left|Q\left(g_{0}\right)\right|=\left|P\left(g_{0}\right)\left(t_{0}\right)\right| \\
& =\left|\operatorname{sgn}\left(\delta_{t_{0}}\left(g_{0}\right)\right) \delta_{t_{0}} P\left(g_{0}\right)\right| \leqslant v(P),
\end{aligned}
$$

which shows that $\|P\|=v(P)$ because $\left(g_{0}, \operatorname{sgn}\left(\delta_{t_{0}}\left(g_{0}\right)\right) \delta_{t_{0}}\right) \in \Pi(C(K))$.

Theorem 2.3. Let $E$ be a complex Banach space. For every positive integer $k$, we have

$$
n^{(k)}(E) \geqslant k^{k /(1-k)}
$$

and the constant $k^{k /(1-k)}$ is sharp.

Proof. By [14, Theorem 1], it is true that $\|P\| \leqslant k^{k /(k-1)} v(P)$ for each $P \in \mathcal{P}\left({ }^{k} E\right.$ : $E)$. This follows from the fact that

$$
v\left(\frac{P}{\|P\|}\right)=\frac{1}{\|P\|} v(P)
$$

and the definition of $n^{(k)}(E)$. In $[\mathbf{1 4}, \S 7]$ it is proved that for every $k \in \mathbb{N}$ there is a two-dimensional space $E$ with $n^{(k)}(E)=k^{k /(1-k)}$.

Lemma 2.4. Let $E$ be a Banach space. Let $P \in \mathcal{P}\left({ }^{k} E: E\right), x \in B_{E}$. For $1 \leqslant m<k$, we have

$$
v\left(\hat{D}^{m} P(x)\right) \leqslant \frac{k^{(k+(k /(k-1)))} m !}{(k-m)^{k-m} m^{m}} v(P),
$$

where $\hat{D}^{m} P(x) \in \mathcal{P}\left({ }^{k-m} E: E\right)$ is defined by $\hat{D}^{m} P(x)(y)=\check{P}\left(x^{m}, y^{k-m}\right)$ for $x, y \in E$.

Proof. By a result of Harris [15, Corollary 3] and Theorem 2.3, it follows that

$$
\begin{aligned}
v\left(\hat{D}^{m} P(x)\right) \leqslant\left\|\hat{D}^{m} P(x)\right\| & \leqslant \frac{k^{k} m !}{(k-m)^{k-m} m^{m}}\|P\| \\
& \leqslant \frac{k^{k} m !}{(k-m)^{k-m} m^{m}} k^{k /(k-1)} v(P) .
\end{aligned}
$$


Proposition 2.5. Let $E$ be a Banach space. For every positive integer $k \geqslant 2$, we have

$$
n^{(k)}(E) \leqslant n^{(k-1)}(E) \leqslant \frac{k^{(k+(1 /(k-1)))}}{(k-1)^{k-1}} n^{(k)}(E) .
$$

Proof. First we will prove the left inequality, $n^{(k)}(E) \leqslant n^{(k-1)}(E)$, for every Banach space $E$ and every $k \geqslant 2$.

Indeed, let $\alpha=n^{(k)}(E)$. Let $Q \in S_{\mathcal{P}\left({ }^{k-1} E: E\right)}$. Let $\left\{x_{i}\right\} \subset S_{E}$ such that $\left\|Q\left(x_{i}\right)\right\| \rightarrow 1$ as $i \rightarrow \infty$. Define $P_{i}(x)=x_{i}^{*}(x) Q(x)$ for $x \in E$, where $x_{i}^{*} \in E^{*}$, with $\left\|x_{i}^{*}\right\|=x_{i}^{*}\left(x_{i}\right)=1$ for every positive integer $i$. Then $P_{i} \in \mathcal{P}\left({ }^{k} E: E\right)$. Note that $\left\|P_{i}\right\| \rightarrow 1$ as $i \rightarrow \infty$. Since $v\left(P_{i} /\left\|P_{i}\right\|\right) \geqslant \alpha$, we have

$$
\begin{aligned}
\alpha\left\|P_{i}\right\| & \leqslant v\left(P_{i}\right) \\
& =\sup _{\left(x, x^{*}\right) \in \Pi(E)}\left|x^{*}\left(P_{i}(x)\right)\right| \\
& =\sup _{\left(x, x^{*}\right) \in \Pi(E)}\left|x_{i}^{*}(x)\right|\left|x^{*}(Q(x))\right| \\
& \leqslant \sup _{\left(x, x^{*}\right) \in \Pi(E)}\left|x^{*}(Q(x))\right| \\
& =v(Q) .
\end{aligned}
$$

Taking the limit as $i \rightarrow \infty$, we get $\alpha \leqslant v(Q)$. Since $Q \in S_{\mathcal{P}\left({ }^{k-1} E: E\right)}$ was arbitrary, we obtain the left inequality.

In order to prove the right inequality, let $P \in S_{\mathcal{P}\left({ }^{k} E: E\right)}, x \in S_{E}$. By Lemma 2.4, it follows that

$$
\begin{aligned}
n^{(k)}(E) & \leqslant n^{(k-1)}(E) \leqslant v\left(\frac{\hat{D} P(x)}{\|\hat{D} P(x)\|}\right)=\frac{1}{\|\hat{D} P(x)\|} v(\hat{D} P(x)) \\
& \leqslant \frac{k^{(k+(k /(k-1)))}}{(k-1)^{k-1}} \frac{v(P)}{\|\hat{D} P(x)\|} .
\end{aligned}
$$

We claim that

$$
\inf _{P \in S_{\mathcal{P}\left(k_{E: E)}\right)}, x \in S_{E}} \frac{v(P)}{\|\hat{D} P(x)\|} \leqslant \frac{1}{k} n^{(k)}(E)
$$

Let

$$
I=\inf _{P \in S_{\mathcal{P}\left(k_{E}: E\right)}, x \in S_{E}} \frac{v(P)}{\|\hat{D} P(x)\|} .
$$

Then

$$
\begin{aligned}
I & =\inf _{P \in S_{\mathcal{P}\left({ }^{k} E: E\right)}}\left\{v(P) \inf _{x \in S_{E}} \frac{1}{\|\hat{D} P(x)\|}\right\} \\
& =\inf _{P \in S_{\mathcal{P}\left(k_{E}: E\right)}}\left\{v(P) \frac{1}{\sup _{x \in S_{E}}\|\hat{D} P(x)\|}\right\} .
\end{aligned}
$$

We show that

$$
\sup _{x \in S_{E}}\|\hat{D} P(x)\| \geqslant k
$$


Indeed,

$$
\begin{aligned}
\sup _{x \in S_{E}}\|\hat{D} P(x)\| & =\sup _{x \in S_{E}}\left(\sup _{y \in S_{E}}\|\hat{D} P(x)(y)\|\right) \\
& =k \sup _{x, y \in S_{E}}\left\|\check{P}\left(x^{k-1} y\right)\right\| \geqslant k \sup _{x \in S_{E}}\|P(x)\|=k .
\end{aligned}
$$

So,

$$
I \leqslant \inf _{P \in S_{\mathcal{P}\left({ }^{k} E: E\right)}}\left\{v(P) \frac{1}{k}\right\}=\frac{1}{k} n^{(k)}(E)
$$

Therefore,

$$
n^{(k)}(E) \leqslant n^{(k-1)}(E) \leqslant \frac{k^{(k+(k /(k-1)))}}{(k-1)^{k-1}} I \leqslant \frac{k^{(k+(1 /(k-1)))}}{(k-1)^{k-1}} n^{(k)}(E) .
$$

Remark 2.6. If $l_{2}$ is a real Hilbert space, then $n^{(k)}\left(l_{2}\right)=0$ for every $k \geqslant 2$.

Remark 2.7. If $l_{2}$ is a complex Hilbert space, then $n^{(k)}\left(l_{2}\right) \leqslant \frac{1}{2}$ for every $k \geqslant 2$.

The proof of the following proposition is almost the same as the one given in $[\mathbf{1 9}$, Proposition 1].

Proposition 2.8. For every Banach space $E_{\lambda}$ and every positive integer $k$, we have

(1) $n^{(k)}\left(\left[\bigoplus_{\lambda \in \Lambda} E_{\lambda}\right]_{c_{0}}\right) \leqslant \inf _{\lambda \in \Lambda} n^{(k)}\left(E_{\lambda}\right)$;

(2) $n^{(k)}\left(\left[\bigoplus_{\lambda \in \Lambda} E_{\lambda}\right]_{l_{1}}\right) \leqslant \inf _{\lambda \in \Lambda} n^{(k)}\left(E_{\lambda}\right)$;

(3) $n^{(k)}\left(\left[\bigoplus_{\lambda \in \Lambda} E_{\lambda}\right]_{l_{\infty}}\right) \leqslant \inf _{\lambda \in \Lambda} n^{(k)}\left(E_{\lambda}\right)$.

Proof. We prove only (2) because the proofs of (1) and (3) are similar. Let $P \in \mathcal{P}\left({ }^{k} X\right.$ : $X)$ with $\|P\|=1$. Let $Q \in \mathcal{P}\left({ }^{k} X \oplus_{1} Y: X \oplus_{1} Y\right)$ be such that $Q(x, y)=(P(x), 0)$. Then $\|Q\|=1$. Given $\varepsilon>0$, there exist $(x, y) \in S_{X \oplus_{1} Y}$ and $\left(x^{*}, y^{*}\right) \in S_{\left(X \oplus_{1} Y\right)^{*}}$ such that $x^{*}(x)+y^{*}(y)=\left\|x^{*}\right\|\|x\|+\left\|y^{*}\right\|\|y\|=1$ and

$$
\begin{aligned}
n^{(k)}\left(X \oplus_{1} Y\right)-\varepsilon & \leqslant\left|\left(x^{*}, y^{*}\right) Q(x, y)\right| \\
& =\left|x^{*}(P(x))\right| \leqslant \frac{1}{\left\|x^{*}\right\|\|x\|^{k}}\left|x^{*}(P(x))\right|=\left|\frac{x^{*}}{\left\|x^{*}\right\|} P\left(\frac{x}{\|x\|}\right)\right| \leqslant v(P),
\end{aligned}
$$

because $\left(1 /\left\|x^{*}\right\|\|x\|^{k}\right) \geqslant 1$. Thus $n^{(k)}\left(X \oplus_{1} Y\right) \leqslant n^{(k)}(X)$. 
In $l_{1}(\mu, \mathbb{R})=l_{1}$, the inequality in Proposition $2.8(2)$ is strict because $n^{(2)}(\mathbb{R})=1$ and $n^{(2)}\left(l_{1}(\mu, \mathbb{R})\right) \leqslant \frac{1}{2}$. Indeed, as in $[\mathbf{5}]$, let $P \in \mathcal{P}\left({ }^{2} l_{1}: l_{1}\right)$ be defined by

$$
P(x)=\left(\frac{1}{2} x_{1}^{2}+2 x_{1} x_{2},-\frac{1}{2} x_{2}^{2}-x_{1} x_{2}, 0,0, \ldots\right) \quad\left(\text { for } x=\left(x_{i}\right) \in l_{1}\right) .
$$

Then it is not difficult to show that $\|P\|=1$ and $v(P)=\frac{1}{2}$. Thus $n^{(2)}\left(l_{1}\right) \leqslant \frac{1}{2}$.

The following lemma can be deduced from Corollary 2 of [14].

Lemma 2.9. Let $K$ be a compact Hausdorff space and let $k$ be a positive integer. Let $Q \in \mathcal{P}\left({ }^{k} C(K, E): C(K, E)\right)$. Then

$$
v(Q)=\sup \left\{\left|x^{*}(Q(f)(t))\right|: f \in S_{C(K, E)}, t \in K, x^{*} \in S_{E^{*}}, x^{*}(f(t))=1\right\} .
$$

The proof of the following theorem is almost the same as the one given in $[\mathbf{1 9}$, Theorem 5].

Proposition 2.10. Let $K$ be a compact Hausdorff space. For every positive integer $k$, we have $n^{(k)}(C(K, E)) \leqslant n^{(k)}(E)$.

Proof. Let $P \in \mathcal{P}\left({ }^{k} E: E\right)$ with $\|P\|=1$. Define $Q \in \mathcal{P}\left({ }^{k} C(K, E): C(K, E)\right)$ by

$$
Q(f)(t)=P(f(t)) \quad(t \in K, f \in C(K, E)) .
$$

Then $\|Q\|=1$. So, $v(Q) \geqslant n^{(k)}(C(K, E))$. By Lemma 2.9, given $\varepsilon>0$, we can find $f \in S_{C(K, E)}, t \in K, x^{*} \in S_{E^{*}}$ such that $x^{*}(f(t))=1$ and

$$
\left|x^{*}(P(f(t)))\right|=\left|x^{*}(Q(f)(t))\right|>n^{(k)}(C(K, E))-\varepsilon .
$$

Thus $n^{(k)}(C(K, E)) \leqslant n^{(k)}(E)$.

Let $E$ and $F$ be Banach spaces. A bounded $k$-homogeneous polynomial $P$ has an extension $\bar{P} \in \mathcal{P}\left({ }^{k} E^{* *}: F^{* *}\right)$ to the bidual $E^{* *}$ of $E$, which is called the Aron-Berner extension of $P$ (see $[\mathbf{1}]$ ). In fact, $\bar{P}$ is defined in the following way. We first start with the complex-valued bounded $k$-homogeneous polynomial $P \in \mathcal{P}\left({ }^{k} E\right)$. Let $A$ be the bounded symmetric $k$-linear form on $E$ corresponding to $P$. We can extend $A$ to an $k$-linear form $\bar{A}$ on the bidual $E^{* *}$ in such a way that, for each fixed $j, 1 \leqslant j \leqslant k$, and, for each fixed $x_{1}, \ldots, x_{j-1} \in E$ and $z_{j+1}, \ldots, z_{m} \in E^{* *}$, the linear form

$$
z \rightarrow \bar{A}\left(x_{1}, \ldots, x_{j-1}, z, z_{j+1}, \ldots, z_{k}\right), \quad z \in E^{* *},
$$

is weak* continuous. By this weak* continuity $A$ can be extended to a $k$-linear form $\bar{A}$ on $E^{* *}$, beginning with the last variable and working backwards to the first. Then the restriction

$$
\bar{P}(z)=\bar{A}(z, \ldots, z)
$$

is called the Aron-Berner extension of $P$. In particular, Davie and Gamelin [9] proved that $\|P\|=\|\bar{P}\|$. It is also worth remarking that $\bar{A}$ is not symmetric in general. 
Next, for a vector-valued $k$-homogeneous polynomial $P \in \mathcal{P}\left({ }^{k} E: F\right)$, the Aron-Berner extension $\bar{P} \in \mathcal{P}\left({ }^{k} E^{* *}: F^{* *}\right)$ is defined as follows: given $z \in E^{* *}$ and $w \in F^{*}$,

$$
\bar{P}(z)(w)=\overline{w \circ P}(z) .
$$

For $x \in E$, we define $\delta_{x}: E^{*} \rightarrow \mathbb{C}$ by $\delta_{x}\left(x^{*}\right)=x^{*}(x)$ for each $x^{*} \in E^{*}$. Then $\delta_{x} \in E^{* *}$.

Let $\left\langle x_{\alpha}\right\rangle$ be a net in $E$ and let $x_{0}^{* *} \in E^{* *}$. We say that $\left\langle x_{\alpha}\right\rangle$ converges polynomial $^{*}$ to $x_{0}^{* *}$ if, for every $P \in \mathcal{P}\left({ }^{k} E\right)(k \in \mathbb{N})$, we have that $P\left(x_{\alpha}\right)$ converges to $\bar{P}\left(x_{0}^{* *}\right)$, where $\bar{P}$ is the Aron-Berner extension of $P$.

A function $f: E^{* *} \rightarrow F^{*}$ is called ( $\left.\operatorname{pol}^{*}, \mathrm{w}^{*}\right)$-continuous if $x_{0}^{* *} \in E^{* *}$ and $\left\langle x_{\alpha}\right\rangle$ is a net in $E$ such that $\left\langle x_{\alpha}\right\rangle$ converges polynomially* to $x_{0}^{* *}$, then $\left\langle f\left(\delta_{x_{\alpha}}\right)\right\rangle$ converges weakly* to $f\left(x_{0}^{* *}\right)$.

The proof of the following theorem is very close to the one given in [4, Theorem 17.2].

Theorem 2.11. Let $E$ be a Banach space. Let $P \in \mathcal{P}\left({ }^{k} E^{* *}: E^{* *}\right)(n \geqslant 1)$ be $\left(\right.$ pol $\left.^{*}, w^{*}\right)$-continuous. Let

$$
L V(P):=\left\{P\left(x^{\prime \prime}\right)\left(x^{\prime}\right):\left(x^{\prime}, x^{\prime \prime}\right) \in \Pi\left(E^{*}\right)\right\}
$$

and

$$
l V(P):=\left\{\delta_{x^{\prime}}\left(P\left(\delta_{x}\right)\right):\left(x, x^{\prime}\right) \in \Pi(E)\right\} .
$$

Then $l V(P) \subset V(P) \subset \overline{l V(P)}$, so $\overline{l V(P)}=\overline{V(P)}$.

Proof. We may assume that $\|P\|=1$. Clearly, $l V(P) \subset V(P)$.

Claim 2.12. $V(P) \subset \overline{L V(P)}$.

Let $\lambda \in V(P)$. Then $\lambda=x_{0}^{\prime \prime \prime}\left(P\left(x_{0}^{\prime \prime}\right)\right)$ for some $\left(x_{0}^{\prime \prime}, x_{0}^{\prime \prime \prime}\right) \in \Pi\left(E^{* *}\right)$. Let $0<\varepsilon<1$. By the uniform continuity of $P$ on $B_{E^{* *}}$ there is a $0<\delta<\frac{1}{3} \varepsilon$ such that, for $x^{\prime \prime}, y^{\prime \prime} \in B_{E^{* *}}$ with $\left\|x^{\prime \prime}-y^{\prime \prime}\right\|<\delta$, we have $\left\|P\left(x^{\prime \prime}\right)-P\left(y^{\prime \prime}\right)\right\|<\frac{1}{3} \varepsilon$. Since $B_{E^{*}}$ is $\mathrm{w}^{*}$-dense in $B_{E^{* * *}}$, there exists $x_{0}^{\prime} \in B_{E^{*}}$ such that

$$
\left|\delta_{x_{0}^{\prime}}\left(P\left(x_{0}^{\prime \prime}\right)\right)-x_{0}^{\prime \prime \prime}\left(P\left(x_{0}^{\prime \prime}\right)\right)\right|=\left|\lambda-\delta_{x_{0}^{\prime}}\left(P\left(x_{0}^{\prime \prime}\right)\right)\right|<\delta
$$

and

$$
\left|\delta_{x_{0}^{\prime}}\left(x_{0}^{\prime \prime}\right)-x_{0}^{\prime \prime \prime}\left(x_{0}^{\prime \prime}\right)\right|=\left|1-x_{0}^{\prime \prime}\left(x_{0}^{\prime}\right)\right|<\frac{1}{4} \delta^{2} .
$$

By the Bishop-Phelps-Bollobas theorem [2] there exist $\left(y_{0}^{\prime}, y_{0}^{\prime \prime}\right) \in \Pi\left(E^{*}\right)$ such that $\left\|x_{0}^{\prime}-y_{0}^{\prime}\right\|<\delta$ and $\left\|x_{0}^{\prime \prime}-y_{0}^{\prime \prime}\right\|<\delta$. Thus $\delta_{y_{0}^{\prime}}\left(P\left(y_{0}^{\prime \prime}\right)\right) \in L V(P)$. It follows that

$$
\begin{aligned}
\mid \lambda-\delta_{y_{0}^{\prime}}( & \left.P\left(y_{0}^{\prime \prime}\right)\right) \mid \\
& \leqslant\left|\lambda-\delta_{x_{0}^{\prime}}\left(P\left(x_{0}^{\prime \prime}\right)\right)\right|+\left|P\left(x_{0}^{\prime \prime}\right)\left(x_{0}^{\prime}\right)-P\left(x_{0}^{\prime \prime}\right)\left(y_{0}^{\prime}\right)\right|+\left|P\left(x_{0}^{\prime \prime}\right)\left(y_{0}^{\prime}\right)-P\left(y_{0}^{\prime \prime}\right)\left(y_{0}^{\prime}\right)\right| \\
& <\delta+\left\|P\left(x_{0}^{\prime \prime}\right)\right\|\left\|x_{0}^{\prime}-y_{0}^{\prime}\right\|+\left\|P\left(x_{0}^{\prime \prime}\right)-P\left(y_{0}^{\prime \prime}\right)\right\| \\
& <3 \delta<\varepsilon,
\end{aligned}
$$

which shows that $\lambda \in \overline{L V(P)}$. Thus $V(P) \subset \overline{L V(P)}$. 
Claim 2.13. $L V(P) \subset \overline{l V(P)}$.

Let $\beta \in L V(P)$. Then $\beta=P\left(x_{0}^{\prime \prime}\right)\left(x_{0}^{\prime}\right)=\delta_{x_{0}^{\prime}}\left(P\left(x_{0}^{\prime \prime}\right)\right)$ for some $\left(x_{0}^{\prime}, x_{0}^{\prime \prime}\right) \in \Pi\left(E^{*}\right)$. Let $0<\varepsilon<1$. By the Davie-Gamelin theorem [9] $\left(B_{E}\right.$ is pol*-dense in $\left.B_{E^{* *}}\right)$ there is a net $\left\langle x_{\alpha}\right\rangle$ in $B_{E}$ such that $\delta_{x_{\alpha}}$ converges pol* to $x_{0}^{\prime \prime}$. Then $\delta_{x_{0}^{\prime}}\left(\delta_{x_{\alpha}}\right)=x_{0}^{\prime}\left(x_{\alpha}\right)$ converges to $\delta_{x_{0}^{\prime}}\left(x_{0}^{\prime \prime}\right)=x_{0}^{\prime \prime}\left(x_{0}^{\prime}\right)=1$. Let $Q=\delta_{x_{0}^{\prime}} \circ P \in \mathcal{P}\left({ }^{k} E^{* *}\right)$. Since $P \in \mathcal{P}\left({ }^{k} E^{* *}: E^{* *}\right)$ is $\left(\right.$ pol $\left.^{*}, \mathrm{w}^{*}\right)$ continuous, $Q\left(\delta_{x_{\alpha}}\right)=\delta_{x_{0}^{\prime}}\left(P\left(\delta_{x_{\alpha}}\right)\right)$ converges to $Q\left(x_{0}^{\prime \prime}\right)=\delta_{x_{0}^{\prime}}\left(P\left(x_{0}^{\prime \prime}\right)\right)=\beta$. Choose $x_{\alpha_{0}}$ such that

$$
\left|\beta-\delta_{x_{0}^{\prime}}\left(P\left(\delta_{x_{\alpha_{0}}}\right)\right)\right|<\delta \text { and }\left|x_{0}^{\prime}\left(x_{\alpha_{0}}\right)-1\right|<\frac{1}{4} \delta^{2} .
$$

By the Bishop-Phelps-Bollobas theorem $[2]$ there is $\left(y_{0}, y_{0}^{\prime}\right) \in \Pi(E)$ such that $\| x_{\alpha_{0}}-$ $y_{0} \|<\delta$ and $\left\|x_{0}^{\prime}-y_{0}^{\prime}\right\|<\delta$. Then $\delta_{y_{0}^{\prime}}\left(P\left(\delta_{y_{0}}\right)\right) \in l V(P)$. We have

$$
\begin{aligned}
\mid \beta- & \delta_{y_{0}^{\prime}}\left(P\left(\delta_{y_{0}}\right)\right) \mid \\
& \leqslant\left|\beta-\delta_{x_{0}^{\prime}}\left(P\left(\delta_{x_{\alpha_{0}}}\right)\right)\right|+\left|\delta_{x_{0}^{\prime}}\left(P\left(\delta_{x_{\alpha_{0}}}\right)\right)-\delta_{x_{0}^{\prime}}\left(P\left(\delta_{y_{0}}\right)\right)\right|+\left|\delta_{x_{0}^{\prime}}\left(P\left(\delta_{y_{0}}\right)\right)-\delta_{y_{0}^{\prime}}\left(P\left(\delta_{y_{0}}\right)\right)\right| \\
& <\delta+\left\|P\left(\delta_{x_{\alpha_{0}}}\right)-P\left(\delta_{y_{0}}\right)\right\|+\left\|P\left(\delta_{y_{0}}\right)\right\|\left\|x_{0}^{\prime}-y_{0}^{\prime}\right\| \\
& <3 \delta<\varepsilon
\end{aligned}
$$

which shows that $\beta \in \overline{l V(P)}$. Thus $L V(P) \subset \overline{l V(P)}$. Thus, by Claims 2.12 and 2.13, $V(P) \subset \overline{l V(P)}$.

Corollary 2.14. Let $E$ be a Banach space and let $k$ be a positive integer. Let $Q \in$ $\mathcal{P}\left({ }^{k} E: E\right)$. Then $\overline{V(Q)}=\overline{V(\bar{Q})}$, where $\bar{Q}$ is the Aron-Berner extension of $Q$.

Proof. Since $\bar{Q}$ is $\left(\right.$ pol $\left.^{*}, \mathrm{w}^{*}\right)$-continuous and $l V(\bar{Q})=V(Q)$, the corollary is proven.

Corollary 2.15. Let $E$ be a Banach space. For every positive integer $k$, we have $n^{(k)}\left(E^{* *}\right) \leqslant n^{(k)}(E)$.

Proof. For every $Q \in \mathcal{P}\left({ }^{k} E: E\right)$ with $\|Q\|=1$ there is the Aron-Berner extension $\bar{Q} \in \mathcal{P}\left({ }^{k} E^{* *}: E^{* *}\right)$ of $Q$. Davie and Gamelin $[\mathbf{9}]$ proved that $\|Q\|=1=\|\bar{Q}\|$ and Corollary 2.14 shows that $v(Q)=v(\bar{Q})$, which proves the corollary.

\section{Numerical radius of a multilinear map and a polynomial on $C(K)$ and the disc algebra}

In [5] the numerical radius of a k-linear mapping $A \in \mathcal{L}\left({ }^{k} E: E\right)$ is defined by

$$
v(A)=\sup \left\{\left|x^{*}\left(A\left(x_{1}, \ldots, x_{k}\right)\right)\right|:\left(x_{1}, \ldots, x_{k}, x^{*}\right) \in \Pi\left(E^{k}\right)\right\},
$$

where

$$
\Pi\left(E^{k}\right)=\left\{\left(x_{1}, \ldots, x_{k}, x^{*}\right):\left\|x_{j}\right\|=\left\|x^{*}\right\|=1=x^{*}\left(x_{j}\right), j=1, \ldots, k\right\} .
$$

Theorem 3.1. Let $K$ be a compact Hausdorff space and let $P \in \mathcal{P}\left({ }^{k} C(K): C(K)\right)$ $(k \in \mathbb{N})$. Then $v(P)=\|P\|$ or $v(\check{P}) \geqslant\|P\|$, where $\check{P}$ is the symmetric $k$-linear map associated with $P$. 
Proof. Let $\varepsilon>0$. Assume that $\|P\|=1$. Then there exist $f_{0} \in C(K)$ with $\left\|f_{0}\right\|=1$ and $t_{0} \in K$ such that $f_{0}\left(t_{0}\right) \neq 0$ and $\left|P\left(f_{0}\right)\left(t_{0}\right)\right|>1-\varepsilon$. Let $U$ be an open neighbourhood of $t_{0}$ with $0 \notin f_{0}(U)$. By Urysohn's lemma there is a continuous function $\pi: K \rightarrow[0,1]$ such that $\pi\left(t_{0}\right)=1, \pi(K-U)=0$. Now define $\psi$ on $K$ by $\psi(t)=0$ when $f_{0}(t)=0$ and

$$
\psi(t)=\frac{f_{0}(t)}{\left|f_{0}(t)\right|} \sqrt{1-\left|f_{0}(t)\right|^{2}} \pi(t)
$$

where $f_{0}(t) \neq 0$. Then $\psi \in C(K)$. Let $g_{0}=f_{0}+\mathrm{i} \psi, h_{0}=f_{0}-\mathrm{i} \psi$, so that $g_{0}, h_{0} \in C(K)$, $f_{0}=\frac{1}{2}\left(g_{0}+h_{0}\right)$ and $\left|g_{0}\left(t_{0}\right)\right|=\left|h_{0}\left(t_{0}\right)\right|=\left\|g_{0}\right\|=\left\|h_{0}\right\|=1$. Note that

$$
\begin{aligned}
1-\varepsilon<\left|P\left(f_{0}\right)\left(t_{0}\right)\right| & \leqslant \frac{1}{2^{k}} \sum_{0 \leqslant j \leqslant k}{ }_{k} C_{j}\left|\check{P}\left(g_{0}^{j} h_{0}^{k-j}\right)\left(t_{0}\right)\right| \\
& =\frac{1}{2^{k}}\left(\left|P\left(g_{0}\right)\left(t_{0}\right)\right|+\left|P\left(h_{0}\right)\left(t_{0}\right)\right|+\sum_{1 \leqslant j \leqslant k-1}{ }_{k} C_{j}\left|\check{P}\left(g_{0}^{j} h_{0}^{k-j}\right)\left(t_{0}\right)\right|\right),
\end{aligned}
$$

where ${ }_{k} C_{j}=k ! /(j !(k-j) !)$. So we have $\left|P\left(g_{0}\right)\left(t_{0}\right)\right|>1-\varepsilon$ or $\left|P\left(h_{0}\right)\left(t_{0}\right)\right|>1-\varepsilon$ or $\left|\check{P}\left(g_{0}^{j} h_{0}^{k-j}\right)\left(t_{0}\right)\right|>1-\varepsilon$ for some $1 \leqslant j \leqslant k-1$. Note that

$$
1-\varepsilon<\left|P\left(g_{0}\right)\left(t_{0}\right)\right|=\left|\operatorname{sgn}\left(g_{0}\left(t_{0}\right)\right) \delta_{t_{0}}\left(P\left(g_{0}\right)\right)\right| \leqslant v(P)
$$

or

$$
1-\varepsilon<\left|P\left(h_{0}\right)\left(t_{0}\right)\right|=\left|\operatorname{sgn}\left(h_{0}\left(t_{0}\right)\right) \delta_{t_{0}}\left(P\left(h_{0}\right)\right)\right| \leqslant v(P)
$$

or

$$
1-\varepsilon<\left|\check{P}\left(g_{0}^{j} h_{0}^{k-j}\right)\left(t_{0}\right)\right|=\left|\delta_{t_{0}}\left(\check{P}\left(\left(\operatorname{sgn}\left(g_{0}\left(t_{0}\right)\right) g_{0}\right)^{j}\left(\operatorname{sgn}\left(h_{0}\left(t_{0}\right)\right) h_{0}\right)^{k-j}\right)\right)\right| \leqslant v(\check{P}),
$$

because

$$
\begin{aligned}
\left(g_{0}, \operatorname{sgn}\left(g_{0}\left(t_{0}\right)\right) \delta_{t_{0}}\right),\left(h_{0}, \operatorname{sgn}\left(h_{0}\left(t_{0}\right)\right) \delta_{t_{0}}\right),\left(\operatorname{sgn}\left(g_{0}\left(t_{0}\right)\right) g_{0}, \delta_{t_{0}}\right),\left(\operatorname{sgn}\left(h_{0}\left(t_{0}\right)\right) h_{0}, \delta_{t_{0}}\right) & \in \Pi(C(K)) .
\end{aligned}
$$

Thus $v(P)>1-\varepsilon$ or $v(\check{P})>1-\varepsilon$. Since $\varepsilon>0$ was arbitrary, we have $v(P)=\|P\|$ or $v(\check{P}) \geqslant\|P\|$.

Theorem 3.2. Let $A_{D}$ be the disc algebra. Let $L \in \mathcal{L}\left({ }^{k} A_{D}: A_{D}\right)(k \in \mathbb{N})$. Then $v(L)=\|L\|$.

Proof. Let $\varepsilon>0$. Assume that $\|L\|=1$. It suffices to prove theorem in the case $n=2$. There exist $f_{1}, f_{2} \in A_{D}$ with $\left\|f_{1}\right\|=\left\|f_{2}\right\|=1$ and such that $\left\|L\left(f_{1}, f_{2}\right)\right\|>1-\varepsilon$. Since $L$ is uniformly continuous on the closed unit ball $B_{A_{D}} \times B_{A_{D}}$, there is a $\delta>0$ such that, for all $f_{i}, g_{i} \in B_{A_{D}}(i=1,2)$ with $\left\|f_{i}-g_{i}\right\|<\delta$, we have $\left\|L\left(f_{1}, f_{2}\right)-L\left(g_{1}, g_{2}\right)\right\|<\varepsilon$. By a theorem of Fischer [12] there exist $\alpha_{1}, \ldots, \alpha_{l}, \beta_{1}, \ldots, \beta_{m}$ with $\alpha_{j} \geqslant 0, \beta_{n} \geqslant 0$,

$$
\sum_{1 \leqslant j \leqslant l} \alpha_{j}=\sum_{1 \leqslant n \leqslant m} \beta_{n}=1,
$$


and finite Blaschke products $g_{1}, \ldots, g_{l}, h_{1}, \ldots, h_{m}$ such that

$$
\left\|f_{1}-\sum_{1 \leqslant j \leqslant l} \alpha_{j} g_{j}\right\|<\delta \text { and }\left\|f_{2}-\sum_{1 \leqslant n \leqslant m} \beta_{n} h_{n}\right\|<\delta .
$$

Clearly,

$$
\left\|L\left(f_{1}, f_{2}\right)-L\left(\sum_{1 \leqslant j \leqslant l} \alpha_{j} g_{j}, \sum_{1 \leqslant n \leqslant m} \beta_{n} h_{n}\right)\right\|<\varepsilon
$$

so

$$
\left\|L\left(\sum_{1 \leqslant j \leqslant l} \alpha_{j} g_{j}, \sum_{1 \leqslant n \leqslant m} \beta_{n} h_{n}\right)\right\|>1-2 \varepsilon .
$$

Choose $z_{0} \in \mathbb{C}$ with $\left|z_{0}\right|=1$ such that

$$
\left|L\left(\sum_{1 \leqslant j \leqslant l} \alpha_{j} g_{j}, \sum_{1 \leqslant n \leqslant m} \beta_{n} h_{n}\right)\left(z_{0}\right)\right|=\left\|L\left(\sum_{1 \leqslant j \leqslant l} \alpha_{j} g_{j}, \sum_{1 \leqslant n \leqslant m} \beta_{n} h_{n}\right)\right\| .
$$

Note that $\left|g_{j}\left(z_{0}\right)\right|=\left|h_{n}\left(z_{0}\right)\right|=1$ for all $j, n$. We have

$$
1-2 \varepsilon<\left|L\left(\sum_{1 \leqslant j \leqslant l} \alpha_{j} g_{j}, \sum_{1 \leqslant n \leqslant m} \beta_{n} h_{k} n\right)\left(z_{0}\right)\right| \leqslant \sum_{1 \leqslant j \leqslant l, 1 \leqslant n \leqslant m} \alpha_{j} \beta_{n}\left|L\left(g_{j}, h_{n}\right)\left(z_{0}\right)\right| .
$$

Since

$$
\sum_{1 \leqslant j \leqslant l, 1 \leqslant n \leqslant m} \alpha_{j} \beta_{n}=\left(\sum_{1 \leqslant j \leqslant l} \alpha_{j}\right)\left(\sum_{1 \leqslant n \leqslant m} \beta_{n}\right)=1,
$$

we have $\left|L\left(g_{j_{0}}, h_{n_{0}}\right)\left(z_{0}\right)\right|>1-2 \varepsilon$ for some $j_{0}, n_{0}$. It follows that

$$
1-2 \varepsilon<\left|L\left(g_{j_{0}}, h_{n_{0}}\right)\left(z_{0}\right)\right|=\left|\delta_{z_{0}} L\left(\overline{g_{j_{0}}\left(z_{0}\right)} g_{j_{0}}, \overline{h_{n_{0}}\left(z_{0}\right)} h_{n_{0}}\right)\right| \leqslant v(L)
$$

because

$$
\left(\overline{g_{j_{0}}\left(z_{0}\right)} g_{j_{0}}, \delta_{z_{0}}\right),\left(\overline{h_{n_{0}}\left(z_{0}\right)} h_{n_{0}}, \delta_{z_{0}}\right) \in \Pi\left(A_{D}\right) .
$$

Thus $v(L)>1-2 \varepsilon$. Since $\varepsilon>0$ was arbitrary, we have $v(L)=\|L\|$.

Theorem 3.3. Let $A_{D}$ be the disc algebra. Let $P \in \mathcal{P}\left({ }^{k} A_{D}: A_{D}\right)(k \in \mathbb{N})$. Then $v(P)=\|P\|$ or $v(\check{P}) \geqslant\|P\|$, where $\check{P}$ is the symmetric $k$-linear map associated with $P$.

Proof. Let $\varepsilon>0$. Assume that $\|P\|=1$. Then there exist $f_{0} \in A_{D}$ with $\left\|f_{0}\right\|=1$ such that $\left\|P\left(f_{0}\right)\right\|>1-\varepsilon$. Since $P$ is uniformly continuous on the closed unit ball $B_{A_{D}}$, there is a $\delta>0$ such that, for all $f, g \in B_{A_{D}}$ with $\|f-g\|<\delta$, we have $\|P(f)-P(g)\|<\varepsilon$. By a theorem of Fischer [12] there exist $\alpha_{1}, \ldots, \alpha_{n}$ with $\alpha_{j} \geqslant 0$,

$$
\sum_{1 \leqslant j \leqslant n} \alpha_{j}=1
$$


and finite Blaschke products $g_{1}, \ldots, g_{n}$ such that

$$
\left\|f_{0}-\sum_{1 \leqslant j \leqslant n} \alpha_{j} g_{j}\right\|<\delta .
$$

Clearly,

$$
\left\|P\left(f_{0}\right)-P\left(\sum_{1 \leqslant j \leqslant n} \alpha_{j} g_{j}\right)\right\|<\varepsilon
$$

so

$$
\left\|P\left(\sum_{1 \leqslant j \leqslant n} \alpha_{j} g_{j}\right)\right\|>1-2 \varepsilon
$$

Choose $z_{0} \in C$ with $\left|z_{0}\right|=1$ such that

$$
\left|P\left(\sum_{1 \leqslant j \leqslant n} \alpha_{j} g_{j}\right)\left(z_{0}\right)\right|=\left\|P\left(\sum_{1 \leqslant j \leqslant n} \alpha_{j} g_{j}\right)\right\| .
$$

Note that $\left|g_{j}\left(z_{0}\right)\right|=1$ for all $j=1, \ldots, n$. We have

$$
\begin{aligned}
1 & -2 \varepsilon \\
& <\left|P\left(\sum_{1 \leqslant j \leqslant n} \alpha_{j} g_{j}\right)\left(z_{0}\right)\right| \\
& \leqslant \sum_{i_{1}+\cdots+i_{l}=k} \frac{k !}{i_{1} ! \cdots i_{l} !}\left|\check{P}\left(\left(\alpha_{i_{1}} g_{1}\right)^{i_{1}} \cdots\left(\alpha_{i_{l}} g_{l}\right)^{i_{l}}\right)\left(z_{0}\right)\right| \\
& =\left(\sum_{1 \leqslant j \leqslant n} \alpha_{j}^{k}\left|P\left(g_{j}\right)\left(z_{0}\right)\right|+\sum_{i_{1}+\cdots+i_{l}=k, i_{j}<k} \frac{k !}{i_{1} ! \cdots i_{l} !} \alpha_{i_{1}}^{i_{1}} \cdots \alpha_{i_{l}}^{i_{l}}\left|\check{P}\left(\left(g_{1}\right)^{i_{1}} \cdots\left(g_{k}\right)^{i_{l}}\right)\left(z_{0}\right)\right|\right) .
\end{aligned}
$$

Since

$$
\sum_{1 \leqslant j \leqslant n} \alpha_{j}^{k}+\sum_{i_{1}+\cdots+i_{k}=l, i_{j}<k} \frac{k !}{i_{1} ! \cdots i_{l} !} \alpha_{i_{1}}^{i_{1}} \cdots \alpha_{i_{l}}^{i_{l}}=\left(i_{1}+\cdots+i_{l}\right)^{k}=1,
$$

we have $\left|P\left(g_{j}\right)\left(z_{0}\right)\right|>1-2 \varepsilon$ for some $j$ or $\left|\check{P}\left(\left(g_{1}\right)^{i_{1}} \ldots\left(g_{k}\right)^{i_{l}}\right)\left(z_{0}\right)\right|$ for some $i_{j}$ with $i_{1}+\cdots+i_{l}=k, i_{j}<k$. It follows that

$$
\begin{aligned}
1-2 \varepsilon & <\left|\check{P}\left(\left(\alpha_{i_{1}} g_{1}\right)^{i_{1}} \cdots\left(\alpha_{i_{l}} g_{l}\right)^{i_{l}}\right)\left(z_{0}\right)\right| \\
& =\left|\delta_{z_{0}}\left(\check{P}\left(\left(\overline{g_{1}\left(z_{0}\right)} g_{1}\right)^{i_{1}} \cdots\left(\overline{g_{l}\left(z_{0}\right)} g_{l}\right)^{i_{l}}\right)\right)\left(z_{0}\right)\right| \leqslant v(\check{P})
\end{aligned}
$$

or

$$
1-2 \varepsilon<\left|P\left(g_{j}\right)\left(z_{0}\right)\right|=\left|\overline{g_{j}\left(z_{0}\right)} \delta_{z_{0}}\left(P\left(g_{j}\right)\right)\right| \leqslant v(P)
$$

because

$$
\left(g_{j}, \overline{g_{j}\left(z_{0}\right)} \delta_{z_{0}}\right),\left(\overline{g_{i_{1}}\left(z_{0}\right)} g_{i_{1}}, \delta_{z_{0}}\right), \ldots,\left(\overline{g_{i_{l}}\left(z_{0}\right)} g_{i_{l}}, \delta_{z_{0}}\right) \in \Pi\left(A_{D}\right) .
$$

Thus $v(P)>1-2 \varepsilon$ or $v(\check{P})>1-2 \varepsilon$. Since $\varepsilon>0$ was arbitrary, we have $v(P)=\|P\|$ or $v(\check{P}) \geqslant\|P\|$. 
Recall that (a complex) $M$-space with order unit is isometrically isomorphic to $C(K)$ for some compact Hausdorff space $K$.

Corollary 3.4. Let $E$ be an $M$-space with order unit. Let $P \in \mathcal{P}\left({ }^{k} E: E\right)(k \in \mathbb{N})$. Then $v(P)=\|P\|$ or $v(\check{P}) \geqslant\|P\|$, where $\check{P}$ is the symmetric $k$-linear map associated with $P$.

Acknowledgements. The corresponding author S.G.K. is supported by the Korea Research Foundation under grant KRF-2002-070-C00006. Y.S.C. is supported by the Korea Research Foundation under grant KRF-2002-070-C00005 made in the program year of 2002. D.G. and M.M. are supported in part by MCYT and FEDER project BFM2002-01423. The authors thank an anonymous referee whose careful reading and suggestions led to a much improved version of this paper.

\section{References}

1. R. Aron and P. Berner, A Hahn-Banach extension theorem for analytic functions, Bull. Soc. Math. France 106 (1978), 3-24.

2. B. Bollobas, An extension to the theorem of Bishop and Phelps, Bull. Lond. Math. Soc. 2 (1970), 181-182.

3. F. F. Bonsall and J. Duncan, Numerical ranges of operators on normed spaces and of elements of normed algebras, London Mathematical Society Lecture Note Series, vol. 2 (Cambridge University Press, 1971).

4. F. F. Bonsall and J. Duncan, Numerical ranges, vol. II, London Mathematical Society Lecture Note Series, vol. 10 (Cambridge University Press, 1973).

5. Y. S. CHOI AND S. G. KIM, Norm or numerical radius attaining multilinear mappings and polynomials, J. Lond. Math. Soc. (2) 54 (1996), 135-147.

6. Y. S. Choi, D. Garcia, S. G. Kim And M. Maestre, Norm or numerical radius attaining mappings on $C(K), J$. Math. Analysis Applic. 295 (2004), 80-96.

7. M. J. Crabi, J. Duncan and C. M. McGregor, Some extremal problems in the theory of numerical ranges, Acta Math. 128 (1972), 123-142.

8. M. J. Crabb, J. DunCAn And C. M. McGregor, Mapping theorems and the numerical radius, Proc. Lond. Math. Soc. (3) 25 (1972), 486-502.

9. A. M. Davie and T. W. Gamelin, A theorem on polynomial-star approximation, Proc. Am. Math. Soc. 106 (1989), 351-356.

10. S. DineEn, Complex analysis on infinite dimensional spaces (Springer, 1999).

11. J. Dunchn, C. M. McGregor, J. D. Pryce and A. J. White, The numerical index of a normed space, J. Lond. Math. Soc. 2 (1970), 481-488.

12. S. Fisher, The convex hull of the finite Blaschke products, Bull. Am. Math. Soc. $\mathbf{7 4}$ (1968), 1128-1129.

13. K. E. Gustafson And D. K. M. RAO, Numerical ranges. The fields of values of linear operators and matrices (Springer, 1997).

14. L. HARRIS, The numerical range of holomorphic functions in Banach spaces, Am. J. Math. 43 (1971), 1005-1019.

15. L. HarRIS, A Bernstein-Markov theorem for normed spaces, J. Math. Analysis Applic. 208 (1997), 476-486.

16. G. Lopez, M. Martin and R. Paya, Real Banach spaces with numerical index 1, Bull. Lond. Math. Soc. 31 (1999), 207-212.

17. G. Lumer, Semi-inner-product spaces, Trans. Am. Math. Soc. 100 (1961), 29-43. 
18. C. M. McGregor, Finite dimensional normed linear spaces with numerical index $1, J$. Lond. Math. Soc. (2) 3 (1971), 717-721.

19. M. Martin And R. PAYA, Numerical index of vector-valued function spaces, Studia Math. 142 (2000), 269-280. 